\title{
Quantitative comparison between experimental measurements and CP-FEM predictions of plastic deformation in a tantalum oligocrystal
}

\author{
Hojun Lim ${ }^{\mathrm{a}, *}$, Jay D. Carroll ${ }^{\mathrm{b}}$, Corbett C. Battaile ${ }^{\mathrm{a}}$, Brad L. Boyce ${ }^{\mathrm{b}}$, Christopher R. Weinberger ${ }^{\mathrm{c}}$ \\ ${ }^{a}$ Department of Computational Materials and Data Science, Sandia National Laboratories, Albuquerque NM, 87185 \\ ${ }^{b}$ Department of Metallurgy and Materials Joining, Sandia National Laboratories, Albuquerque NM, 87185 \\ ${ }^{c}$ Department of Mechanical Engineering and Mechanics, Drexel University, Philadelphia, PA, 19104
}

\begin{abstract}
Quantitative comparisons of experimental measurements and model predictions are crucial to validate and improve material models as well as to understand underlying physical phenomena. In this work, we used a recently-developed in situ technique combining high resolution digital image correlation (HR-DIC) and electron backscatter diffraction (EBSD) to obtain intergranular surface strain fields and crystal rotations. These measurements were compared to predictions from a crystal plasticity - finite element method (CP-FEM) simulation. To accurately reproduce the initial grain morphology in the CP-FEM simulation without assumptions regarding the sub-surface microstructure, a coarse columnar-grained tantalum specimen was used. Experimental data was projected onto the finite element mesh to compare measured and simulated data on a point-wise basis. It is shown that model predictions of both surface strain fields and crystal rotations agree reasonably well with HR-DIC and EBSD measurements at various applied strains. This procedure provides an objective and quantitative methodology to evaluate the model-experiment agreement and allows for testing of various model parameters to improve the model.
\end{abstract}

Keywords: Tantalum, crystal plasticity, strain field, texture, EBSD, DIC

\section{Introduction}

Crystal plasticity - finite element method (CP-FEM) models are now widely used to model grain-scale plastic deformation of polycrystalline metals $[1,2,3,4]$. CP-FEM models partition plastic deformation on active slip systems and use a continuum finite element framework to satisfy the strain compatibility and stress equilibrium. Many variations of CP-FEM models have successfully reproduced complex mechanical behavior characteristics of polycrystalline materials, i.e. plastic anisotropy from texture evolution $[5,6,7,8]$. In these models, the macroscopic response of polycrystals is dictated by the response and interaction of individual grains, represented by a single or many finite elements. These polycrystalline CP-FEM models typically construct microstructures by assigning random grain orientations to represent isotropic materials or texture is informed from experimental measurements, i.e. electron backscatter diffraction (EBSD) or X-ray diffraction (XRD) techniques. However, it is difficult to reproduce an accurate three-dimensional initial microstructure without assumptions on sub-surface crystal orientations, grain boundaries and grain shapes. This limitation hinders quantitative comparisons between model predictions and experimentally measured quantities.

\footnotetext{
*Corresponding author

Email address: hnlim@sandia.gov (Hojun Lim)
} 
To avoid this limitation attributed to the unknown subsurface effects of polycrystals, simple structured specimens having a single crystal or two-dimensional columnar grains are often used to compare model predictions with experimental measurements $[9,10,11,12,13,14,15,16,17,18,19$, 20]. In particular, thin tensile specimens having 10 - 20 columnar grains in the gage section, often referred to as 'oligocrystals', are frequently used to assess experiment-model comparisons. Twodimensional columnar grains in oligocrystal specimens conveniently eliminate effects of unknown subsurface microstructure and allow an accurate modeling of the full initial grain morphology. In addition, large grain sizes, on the order of $1 \mathrm{~mm}$, mitigate local grain boundary effects.

Although previous model predictions of surface strain and crystal rotation fields showed qualitative agreement with measured data, quantitative model-experiment comparisons are still lacking, particularly in BCC metals. In this work, direct comparisons between model predictions and experimental measurements were conducted using a tantalum oligocrystal specimen based on previous work by Lim et al. [21]. In particular, we compared measured and predicted data on a point-wise basis to validate the model quantitatively. Using this method, effects of different hardening models that are commonly used in CP-FEM models were investigated.

\section{Experimental Procedure}

A rolled tantalum plate (thickness $=0.8 \mathrm{~mm}$ ) of $99.9 \%$ purity was obtained from Goodfellow Corporation. Using electro-discharge machining (EDM), tensile samples were machined with the tensile axis aligned with the rolling direction (RD) of the plate. The resulting hourglass-shaped specimen had a total length of $23 \mathrm{~mm}$, a gage length of $13.4 \mathrm{~mm}$, and were approximately $1.5 \mathrm{~mm}$ wide at the center (refer to $[21,22]$ for more details). Specimens were heat treated at $2000^{\circ} \mathrm{C}$ for 10 hours at a pressure of $1.33 \times 10^{4} \mathrm{~Pa}\left(10^{-6}\right.$ Torr $)$ in a vacuum furnace to create a columnar microstructure of millimeter-scale grains (i.e. the oligocrystal microstructure) and then polished to a surface finish suitable for EBSD [23]. EBSD measurements were obtained using Channel 5 software (Oxford Instruments) inside a Zeiss Supra 55VP field emission scanning electron microscope (SEM) over a region of interest in the gage section of the specimen of $5.5 \mathrm{~mm} \times 1.4 \mathrm{~mm}$ with a step size of $4 \mu \mathrm{m}$.

Table 1: Average initial crystal orientations obtained from EBSD measurements (Bunge Euler angles in degrees).

\begin{tabular}{cccc|cccc}
\hline Grain & $\phi_{1}$ & $\Phi$ & $\phi_{2}$ & Grain & $\phi_{1}$ & $\Phi$ & $\phi_{2}$ \\
\hline 1 & 130.1 & 44.4 & 46.5 & 7 & 73.2 & 42.8 & 36.2 \\
2 & 99.6 & 51.3 & 45.1 & 8 & 119.0 & 53.0 & 47.5 \\
3 & 61.1 & 44.8 & 35.2 & 9 & 46.7 & 52.4 & 49.6 \\
4 & 72.1 & 15.4 & 23.7 & 10 & 22.6 & 11.6 & 82.0 \\
5 & 76.7 & 38.7 & 47.7 & 11 & 23.7 & 33.4 & 51.0 \\
6 & 46.3 & 23.9 & 56.3 & 12 & 79.7 & 27.2 & 42.3 \\
\hline
\end{tabular}

Figures 1 (a) and (b) show a surface image of the specimen and the initial average crystal orientations with respect to the loading axis, respectively. A total of 12 grains were identified within the region of interest using a $5^{\circ}$ grain boundary misorientation threshold. The average initial crystal orientations are listed in Table 1 in Bunge Euler angles (degrees). The initial grain orientations were scattered within the stereographic triangle such that no significant texture was observed. Figure 1 (c) shows the maximum Schmid factors calculated for twenty four $\{110\}\langle 111\rangle$ slip systems. Note that the Schmid factor, $m$, is defined by $m=\cos \phi \cos \chi$ where $\phi$ is the angle between the slip direction and the loading axis and $\chi$ is the angle between the slip plane normal 

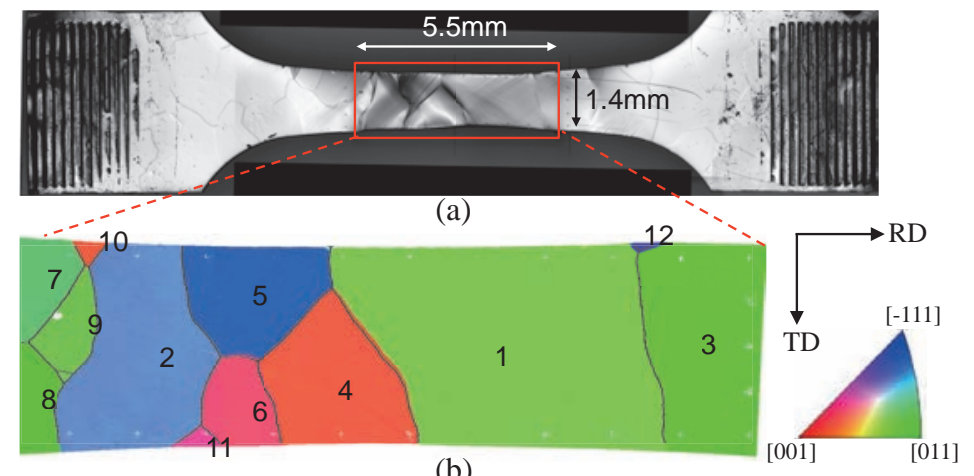

(b)

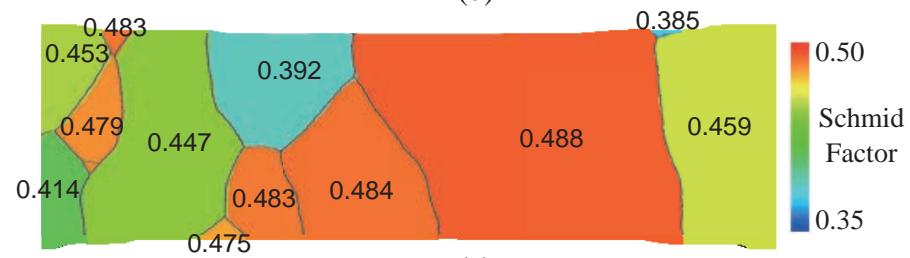

(c)

Figure 1: (a) An optical micrograph of the tantalum oligocrystal tensile specimen, (b) initial grain orientations within the region of interest with respect to the loading direction and (c) the maximum Schmid factors of each grain using $24\{110\}\langle 111\rangle$ slip systems.

and the loading axis. The maximum Schmid factors of grains within gage section of the oligocrystal range between 0.39 (grain 12) and 0.49 (grain 1), representing the hardest and the softest grains, respectively.

After the grain structure on the front surface of the specimen was mapped with EBSD, the specimen was deformed using a custom built in situ load frame inside a scanning electron microscope (SEM) [24, 25]. A uniaxial tension test, interrupted at various strain levels, was performed at a nominal strain rate of $10^{-3} \mathrm{~s}^{-1}$ up to $10 \%$ applied strain as shown in Figure 2. HR-DIC images were captured at each strain level: $0,2,4,6,8$, and $10 \%$ corresponding to points A to F in Figure 2. The DIC speckle pattern consisted of $300 \mathrm{~nm} \mathrm{Cu}$ powder, and the full field results were obtained by stitching DIC results from 12 image locations $(2 \times 6)$ at each strain level. Detailed descriptions of the HR-DIC experimental procedure can be found in the work by Carroll et al. [22, 26]. The image scale for these measurements was $0.439 \mu \mathrm{m} /$ pix (with individual images having a resolution of $3,072 \times 2,304)$. DIC results were obtained using Vic2d software (Correlated Solutions, Inc.) with a subset size of $81 \times 81$ pixels $(36 \times 36 \mu \mathrm{m})$, a step size of 8 pixels $(3.5 \mu \mathrm{m})$, and a strain window of $5 \times 5$ data points. The DIC dataset contained 781,015 points; EBSD measurement points were projected onto the DIC grid (using nearest neighbor interpolation) so that each point in the resulting dataset contained both DIC and EBSD data. The EBSD dataset had a comparable number of points $(\sim 782,000)$ so the amount of over or undersampling was minor. Lagrangian strain fields, $\varepsilon_{x x}, \varepsilon_{y y}$, and $\varepsilon_{x y}$, suitable for a large deformation, were used. Lagrangian finite strain tensor is defined as follows:

$$
\varepsilon_{i j}=\frac{1}{2}\left(u_{i, j}+u_{j, i}+u_{k, i} u_{k, i}\right),
$$

where $u$ is the displacement field.

After the specimen had been loaded to $10 \%$ strain, it was removed from the load frame and 


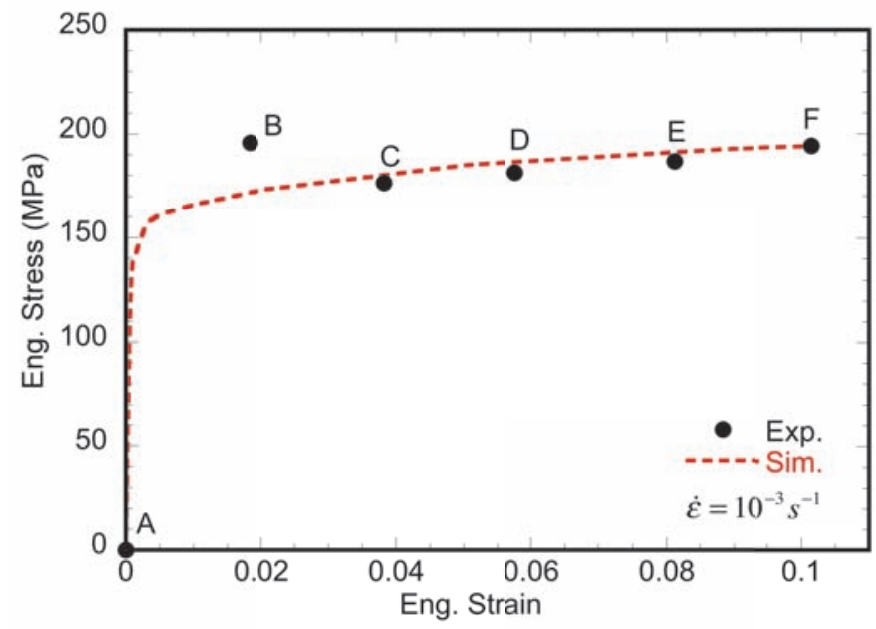

Figure 2: Measured and fitted stress-strain data for the tantalum oligocrystal specimen.

EBSD was performed on the deformed specimen to measure crystal rotations due to loading. Finally, HR-DIC and (initial) EBSD measurements were spatially aligned using fiducial markers.

\section{CP-FEM Simulations}

A BCC crystal plasticity finite element model, developed at Sandia National Laboratories [27, 28], was used to simulate plastic deformation of the tantalum oligocrystal. The model is based on a well-established continuum formulation and follows multiplicative decomposition of the deformation gradient $[1,29,30,31]$. Assuming plastic deformation is caused by dislocation slip, the plastic part of the velocity gradient, $\mathbf{L}_{\mathrm{p}}$, can be written as [1]:

$$
\mathbf{L}_{\mathrm{p}}=\sum_{\alpha} \dot{\gamma}^{\alpha} \mathbf{s}_{0}^{\alpha} \otimes \mathbf{n}_{0}^{\alpha}
$$

where $\mathbf{s}_{0}^{\alpha}$ and $\mathbf{n}_{0}^{\alpha}$ are the initial slip direction and the slip plane normal direction on slip system $\alpha$, respectively. In this work, twenty four $\{110\}\langle 111\rangle$ BCC slip systems were used [21]. The slip rate on the $\alpha$-th slip system, $\dot{\gamma}^{\alpha}$, is represented as a power-law function of resolved shear stress, $\tau^{\alpha}$ and slip resistance, $g^{\alpha}[32]$ :

$$
\dot{\gamma}^{\alpha}=\dot{\gamma}_{0}\left(\frac{\tau^{\alpha}}{g^{\alpha}}\right)^{1 / m},
$$

where, $\dot{\gamma}_{0}$ is the reference shear rate and $m$ is the rate sensitivity factor. The constant values of $\dot{\gamma}_{0}=0.012 \mathrm{~s}^{-1}$ and $m=0.04$ were used in the simulation [21,33]. The slip resistance of the $\alpha$-th slip system, $g^{\alpha}$, in BCC metals can be written as follows, [34]:

$$
g^{\alpha}=\min \left(\tau_{E I}^{* \alpha}, \tau_{L T}^{* \alpha}\right)+\tau_{\text {obs }}
$$

Here, the first term represents the temperature and strain rate dependent lattice resistance based on the dislocation kink-pair theory [28] while $\tau_{\text {obs }}$ is the strain hardening term, attributed to forest dislocations. $\tau_{E I}^{* \alpha}$ and $\tau_{L T}^{* \alpha}$ in Equation (4) denote lattice resistances or thermal parts of the slip resistance at different temperature/ stress regimes calculated from the dislocation elastic interaction 
(EI) model (high temperature and low stress regime) and the dislocation line tension (LT) model (low temperature and high stress regime), respectively $[28,35,36,37,38] . \tau_{E I}^{* \alpha}$ and $\tau_{L T}^{* \alpha}$ can be calculated as follows [28]:

$$
\begin{gathered}
\tau_{E I}^{* \alpha}=\tau_{E I}^{0}\left(1-\frac{T}{T_{c}^{\alpha}(\dot{\gamma})}\right)^{2} \\
\tau_{L T}^{* \alpha}=\tau_{L T}^{0}\left(1-\left(\frac{T}{T_{c}^{\alpha}(\dot{\gamma})}\right)^{1 / 2}\right)
\end{gathered}
$$

Here, $T$ is the temperature, $\tau_{E I}^{0}$ and $\tau_{L T}^{0}$ are material parameters and $T_{c}^{\alpha}(\dot{\gamma})$ is the critical temperature, defined as follows:

$$
T_{c}^{\alpha}(\dot{\gamma})=\frac{2 H_{k}}{k_{\mathrm{B}} \ln \left(\dot{\varepsilon}_{0} / \dot{\gamma}^{\alpha}\right)}
$$

In this equation, $H_{k}$ is the formation enthalpy of an isolated kink, $k_{\mathrm{B}}$ is Boltzmann's constant and $\dot{\varepsilon}_{0}$ is the reference shear rate that relates the temperature and shear strain rate to $H_{k}$ in an Arrhenius expression. Material parameters in Equations (5) - (7) were fit to tantalum single crystal experiments $[39,28]$. The best-fit parameters are listed in Table 2.

The athermal part of the flow stress or the strain hardening term, $\tau_{\text {obs }}$, is formulated using a dislocation density-based Taylor hardening law as follows [40]:

$$
\tau_{\mathrm{obs}}=A \mu b \sqrt{\sum_{\beta=1}^{24} \rho^{\beta}},
$$

where, $A$ is a material constant, $\mu$ is the shear modulus, $b$ is the Burger's vector, and $\rho^{\beta}$ is the dislocation density on slip system $\beta$. The evolution of dislocation density for the $\alpha$-th slip system is obtained by a standard phenomenological equation as follows [41]:

$$
\dot{\rho}^{\alpha}=\left(\kappa_{1} \sqrt{\sum_{\beta=1}^{24} \rho^{\beta}}-\kappa_{2} \rho^{\alpha}\right) \cdot\left|\dot{\gamma}^{\alpha}\right|,
$$

where, $\kappa_{1}$ and $\kappa_{2}$ are hardening parameters representing generation and annihilation of dislocations, respectively and determine the shape of the stress-strain curve. Hardening parameters, $\kappa_{1}$ and $\kappa_{2}$, were determined from least-squares fitting to experimentally-measured tensile data (Points A - F in Figure 2). The best-fit hardening parameters were $\kappa_{1}=1.4 \times 10^{6} \mathrm{~m}^{-1}$ and $\kappa_{2}=15$. The best-fit parameters used in the model are listed in Table 2.

The entire gage section of the tantalum oligocrystal tensile specimen was meshed with 2,140,020 hexahedral elements as shown in Figure 3 (a). To accurately capture the through-thickness stress and strain distributions and to maintain good aspect ratios of finite elements, 60 elements were used through the thickness of the specimen. Note that the total number of experimental data points is approximately 781,015 while there are 35,667 surface elements in the model. Thus, the step size of the experimental data is smaller than the finite element mesh by a factor of 5 . The crystallographic orientation of each finite element in the simulation was determined by superimposing the mesh onto the EBSD map. Each surface element was assigned with the grain orientation corresponding to the 


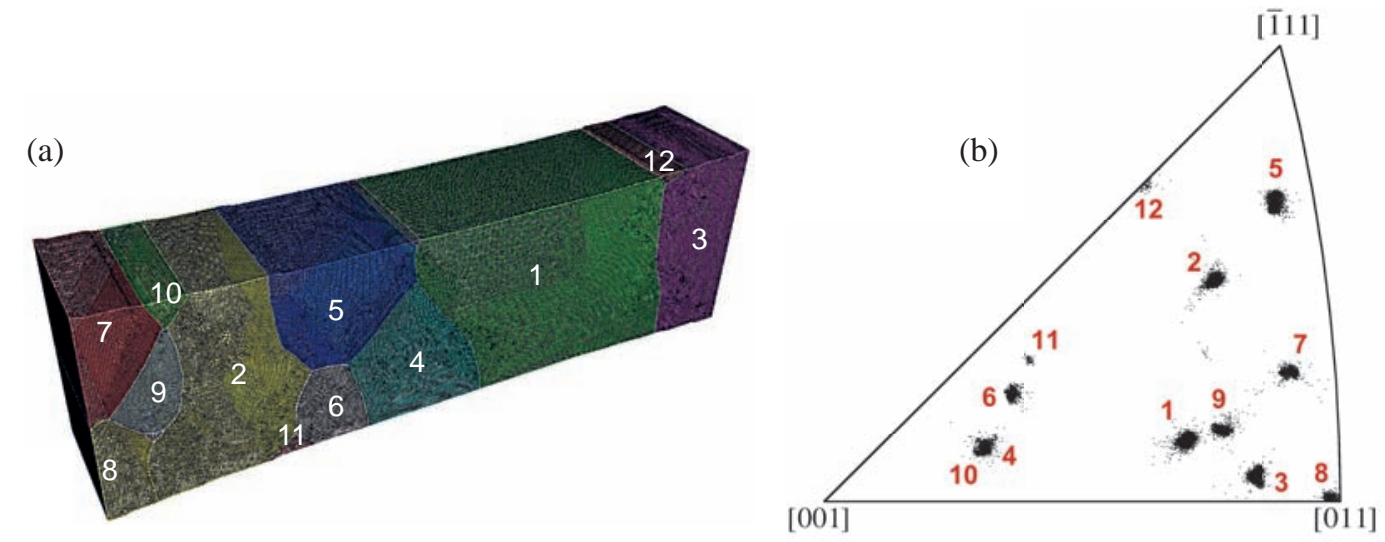

Figure 3: (a) The finite element mesh of the oligocrystal specimen. A total of 2,140,020 hexahedral elements were used. (b) Initial crystal orientation of the oligocrystal specimen with respect to the loading direction.

Table 2: Best-fit parameters, anisotropic elastic constants and shear modulus for tantalum used in the model [42, 28].

\begin{tabular}{cc|cc}
\hline \multicolumn{2}{c|}{ Parameters } & \multicolumn{2}{c}{ Parameters } \\
\hline$\tau_{E I}^{0}$ & $320 \mathrm{MPa}$ & $C_{11}$ & $267 \mathrm{GPa}$ \\
$\tau_{L T}^{0}$ & $406 \mathrm{MPa}$ & $C_{12}$ & $161 \mathrm{GPa}$ \\
$2 H_{k}$ & $0.85 \mathrm{eV}$ & $C_{44}$ & $83 \mathrm{GPa}$ \\
$\dot{\varepsilon}_{0}$ & $2.99 \times 10^{6} \mathrm{~s}^{-1}$ & $\mu$ & $72 \mathrm{GPa}$ \\
$\kappa_{1}$ & $1.4 \times 10^{6} \mathrm{~m}^{6}$ & $A$ & 0.4 \\
$\kappa_{2}$ & 15 & $b$ & $2.87 \AA$ \\
\hline
\end{tabular}

nearest EBSD pixel [21] and the identical grain orientation was assigned to elements through the thickness. The initial spread of orientation within the gage section of the oligocrystal specimen, with respect to the loading direction, is shown in Figure 3 (b). Note that this initial spread may contain both actual orientation scatter as well as experimental error (variability in angular measurements from EBSD). It is difficult to separate these two contributions; thus measured EBSD data is assigned to the model without considering possible measurement error.

\section{Results and Discussion}

\subsection{Comparisons of Strain Fields}

Using the CP-FEM model described in the previous section, uniaxial tension of a tantalum oligocrystal was simulated. As shown in Figure 4, the measured and predicted deformed shapes of the oligocrystal at $10 \%$ applied strain agreed reasonably well, i.e. notable necking was observed in grain 1 for both experiments and model predictions.

Figures $5-7$ compare measured and simulated surface Lagrangian strain fields, $\varepsilon_{x x}, \varepsilon_{y y}$ and $\varepsilon_{x y}$, respectively, at five applied strains $\left(\varepsilon_{a}=2,4,6,8\right.$ and $\left.10 \%\right)$. The CP-FEM model qualitatively captures the heterogeneous intergranular surface strain fields observed in HR-DIC measurements including the locations of localized strain fields at different applied strains. As shown in Figures 5 and 6 , large tensile $\varepsilon_{x x}$ and compressive $\varepsilon_{y y}$ strains were observed in grains 1 and 4 while grain 5 showed less deformation, in accordance with the maximum Schmid factor calculations in Figure 1 (c). Furthermore, the model accurately predicted large shear strains, $\varepsilon_{x y}$, in grains 5 and 6 and 

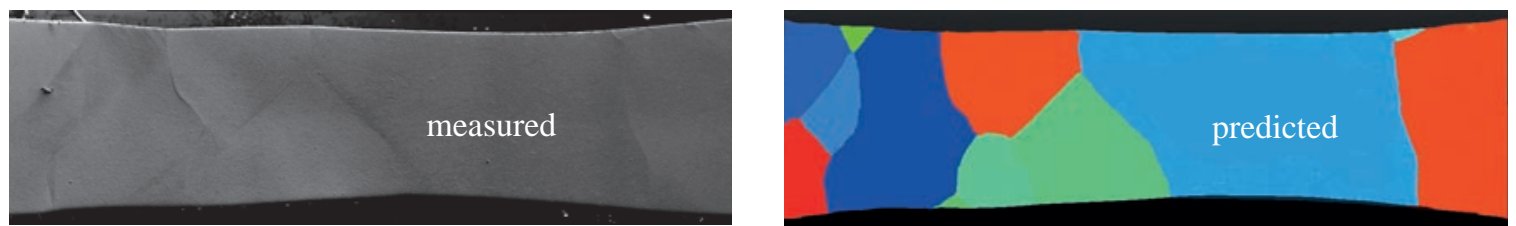

Figure 4: Measured and simulated deformed specimen shapes at $10 \%$ applied strain.
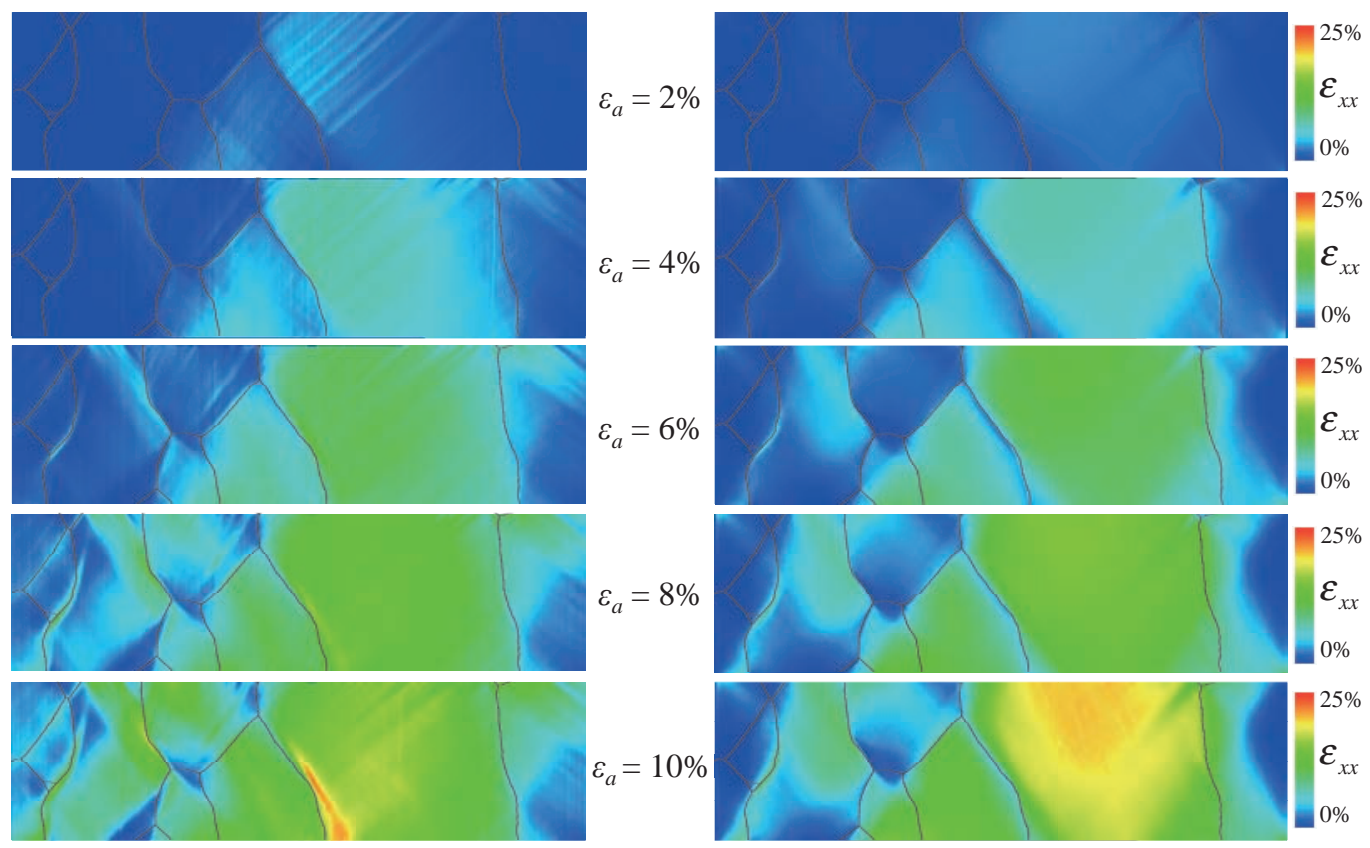

(a) HR-DIC measurements

(b) CP-FEM predictions

Figure 5: A comparison of measured (HR-DIC) and simulated (CP-FEM) $\varepsilon_{x x}$ at applied strains $\left(\varepsilon_{a}\right)$ of $2 \%$ (Point B), $4 \%$ (Point C), $6 \%$ (Point D), $8 \%$ (Point E) and $10 \%$ (Point F).

near the grain boundaries associated with grains 2-6, 2-8, and 2-9. However, some detailed features observed in DIC measurements, i.e. slip lines (at $\varepsilon_{a}=2 \%$ ) and localized strain fields between grains 1 and 4 (at $\varepsilon_{a}=10 \%$ ), were not captured with the model.

To quantitatively compare measured and predicted surface strain fields, the DIC data $(781,015$ data points) was imposed on to the finite element mesh (35,667 surface elements), similar to the procedure used to assign the initial EBSD data to the model. By assigning DIC data on surface finite elements used in the simulation, the experimental measurement and model predictions can be objectively compared on a point-wise basis. In this procedure, the size of the DIC data was reduced by a factor of 22. Figures 8 (a) and (b) show $\varepsilon_{x x}$ at $10 \%$ applied strain from the full HR-DIC data and reduced data, respectively. As shown in Figure 8, this reduction appears to have relatively small effect on the resolution of the strain field. This may indicate that DIC resolution was more than sufficient to resolve the observed strain localizations and that a lower resolution would have been adequate, especially for comparisons with continuum-scale model predictions.

Using HR-DIC measurements and CP-FEM predictions of strain fields with the same data 

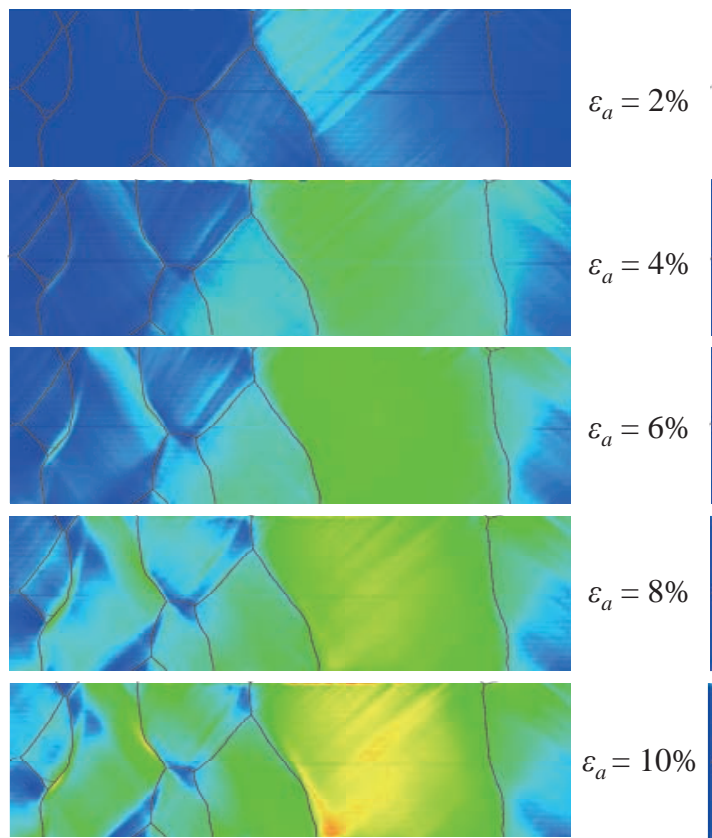

(a) HR-DIC measurements

$\varepsilon_{\mathrm{a}}=10 \%$
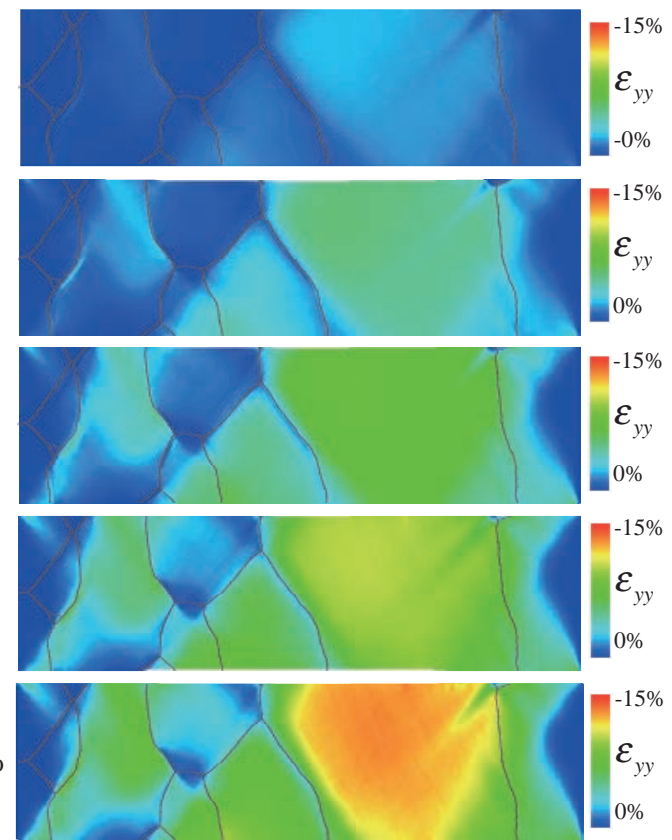

(b) CP-FEM predictions

Figure 6: A comparison of measured (HR-DIC) and simulated (CP-FEM) $\varepsilon_{y y}$ at applied strains $\left(\varepsilon_{a}\right)$ of $2 \%$ (Point B), $4 \%$ (Point C), $6 \%$ (Point D), $8 \%$ (Point E) and $10 \%$ (Point F).
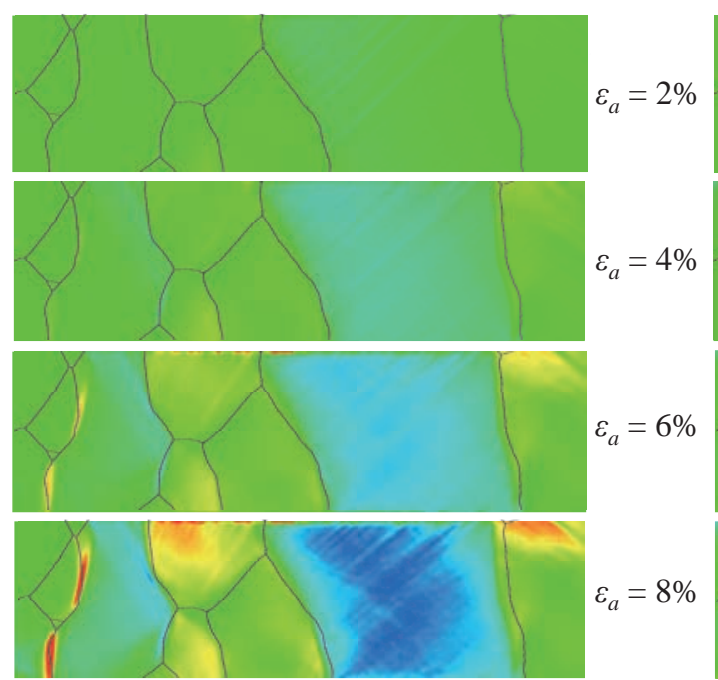

$\varepsilon_{\mathrm{a}}=6 \%$

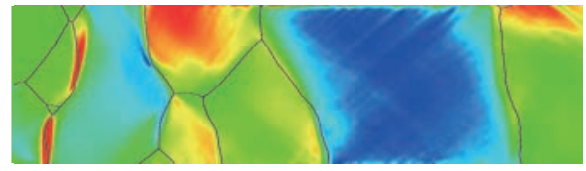

(a) HR-DIC measurements
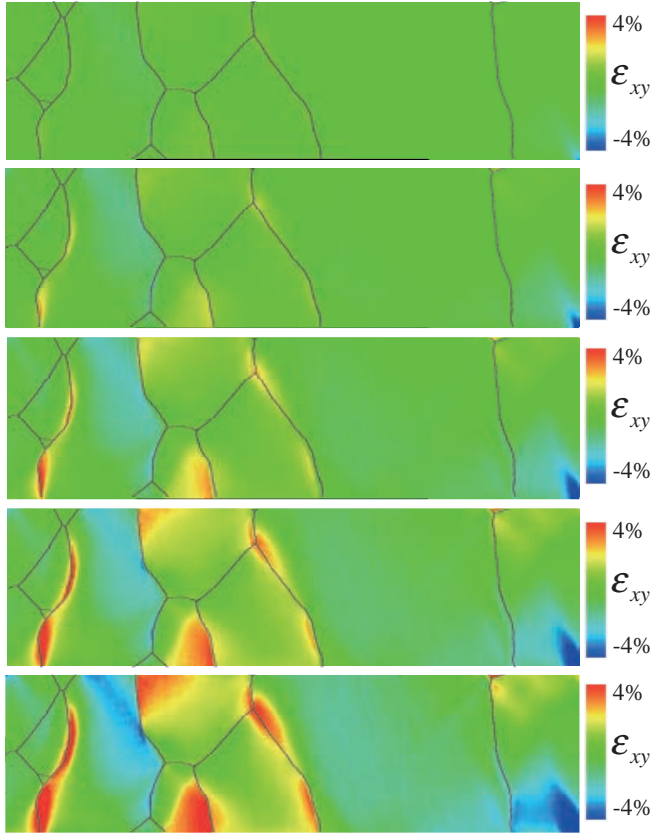

(b) CP-FEM predictions

Figure 7: A comparison of measured (HR-DIC) and simulated (CP-FEM) $\varepsilon_{x y}$ at applied strains $\left(\varepsilon_{a}\right)$ of $2 \%$ (Point B), $4 \%$ (Point C), $6 \%$ (Point D), $8 \%$ (Point E) and $10 \%$ (Point F). 

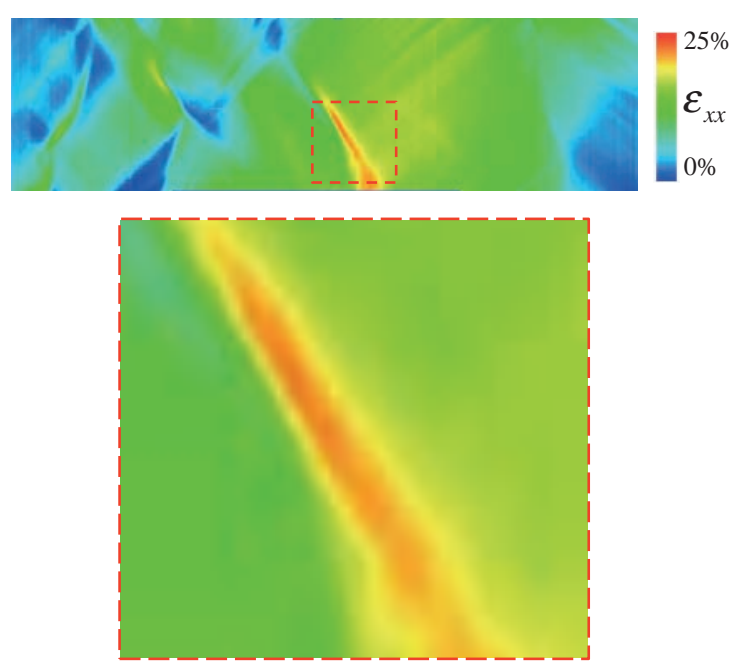

(a) Full DIC data
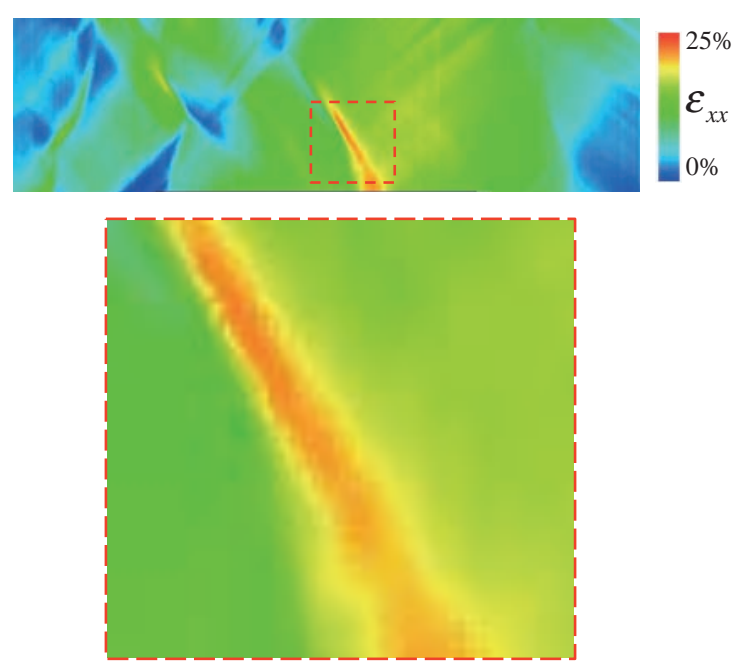

(b) Reduced DIC data

Figure 8: Plots of $\varepsilon_{x x}$ at $10 \%$ applied strain using (a) full HR-DIC data (781,015 data points) and (b) reduced DIC data, imposed on to finite element mesh (35,667 data points).

resolution, deviations between two data sets were obtained as follows:

$$
\Delta \varepsilon=\varepsilon^{\mathrm{DIC}}-\varepsilon^{\mathrm{sim}}
$$

where. $\varepsilon^{\mathrm{DIC}}$ and $\varepsilon^{\mathrm{sim}}$ represent surface strain fields obtained from DIC and CP-FEM simulations, respectively, for each element. Figure 9 shows calculated $\Delta \varepsilon_{x x}$ maps at different applied strain levels. Positive values (red) represent regions where simulation under-predicts strain fields while negative values (blue) show over-predicted regions. It is shown that large $\Delta \varepsilon_{x x}$ values were observed near the grain boundaries and locations where slip lines developed in the experiment, especially near grain boundary $1-4$.

To further quantify the deviation between the measured and predicted strain fields, the quantity $\Delta \varepsilon_{\text {avg }}$, defined as follows, was calculated for each strain component within the entire region.

$$
\Delta \varepsilon^{a v g}=\sqrt{\frac{1}{N} \sum_{i=1}^{N}\left(\varepsilon_{i}^{\mathrm{DIC}}-\varepsilon_{i}^{\mathrm{sim}}\right)^{2}} .
$$

Here, $N$ is the total number of data points $(N=35,667)$. Table 3 lists the average deviation between measured and simulated strain fields at various applied strains. The model agreed relatively well with HR-DIC measurement, i.e. average deviation between the model and experiment at $10 \%$ deformation for $\varepsilon_{x x}$ was around $3 \%$ strain.

Table 3 lists the relative error, $\Delta \varepsilon^{a v g} / \varepsilon_{a}$ at all five applied strain levels within the entire specimen. Here, we chose to normalize $\Delta \varepsilon^{a v g}$ with $\varepsilon_{a}$ instead of $\varepsilon^{\mathrm{DIC}}$ or $\varepsilon^{\text {sim }}$ per data point to objectively compare deviations at different applied strains and because many $\varepsilon^{\text {DIC }}$ and $\varepsilon^{\text {sim }}$ values were very close to zero. An average value of $\Delta \varepsilon_{x x}^{a v g} / \varepsilon_{a}$ for the whole specimen over five applied strains were 0.29. That is, the difference between model predictions and experimental measurements of $\varepsilon_{x x}$ is around $30 \%$ of the applied strain. This value could be used as an objective criterion for comparing different models or for investigating effects of model parameters. Note that $\Delta \varepsilon_{x x}^{a v g} / \varepsilon_{a}$ is fairly 


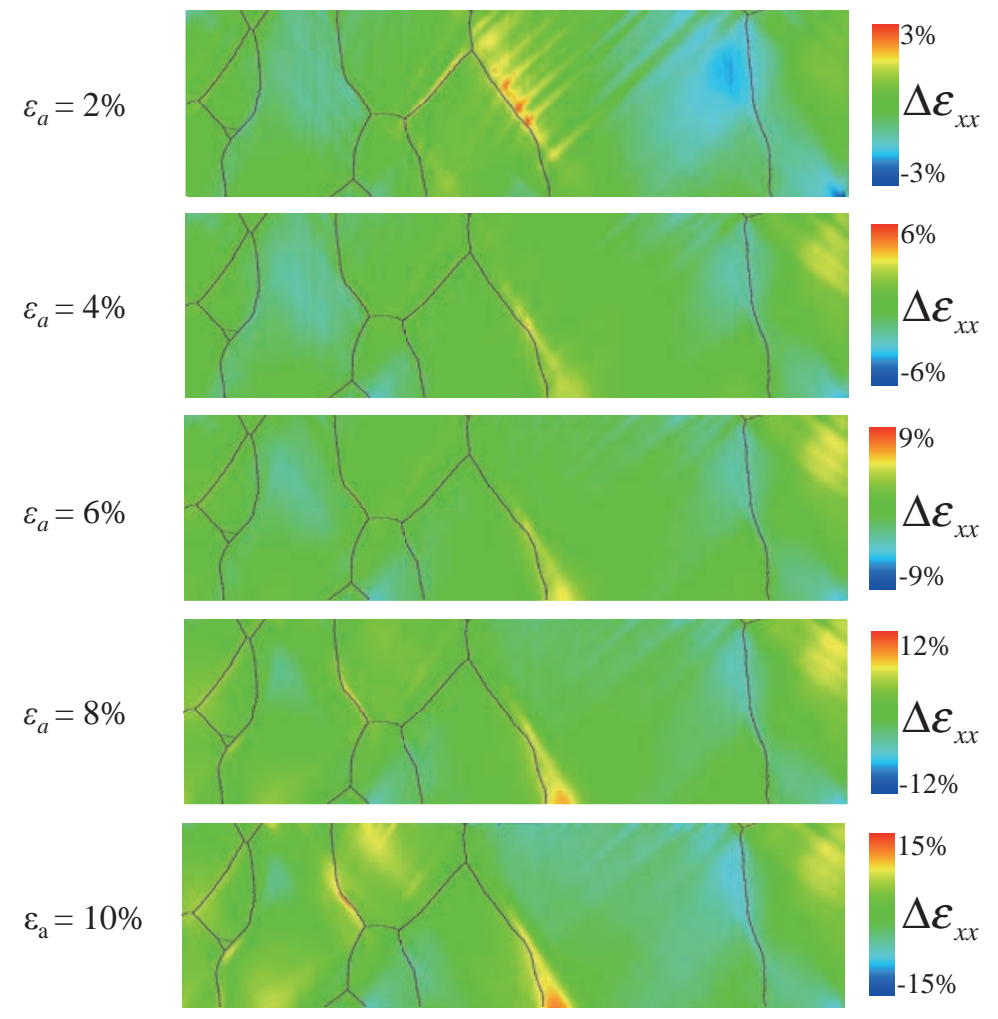

Figure 9: Deviations between measured and simulated surface strain fields, $\varepsilon_{x x}$, at different applied strains. Here, deviations of strain fields are calculated as $\Delta \varepsilon_{x x}=\varepsilon_{x x}^{\mathrm{DIC}}-\varepsilon_{x x}^{\text {sim }}$.

Table 3: Average deviation between measured and simulated strain fields $\left(\Delta \varepsilon^{a v g}\right)$ and relative errors $\left(\Delta \varepsilon^{a v g} / \varepsilon_{a}\right)$ at various applied strains within the whole specimen.

\begin{tabular}{c|ccccc}
\hline & \multicolumn{5}{|c}{ Applied strain $\left(\varepsilon_{a}\right)$} \\
& $2 \%$ & $4 \%$ & $6 \%$ & $8 \%$ & $10 \%$ \\
\hline$\Delta \varepsilon_{x x}^{a v g}$ & 0.0073 & 0.0102 & 0.0143 & 0.0215 & 0.0344 \\
$\Delta \varepsilon_{y y}^{a v g}$ & 0.0059 & 0.0097 & 0.0116 & 0.0142 & 0.0180 \\
$\Delta \varepsilon_{x y}^{a v g}$ & 0.0031 & 0.0071 & 0.0104 & 0.0136 & 0.0159 \\
\hline$\Delta \varepsilon_{x x}^{a v g} / \varepsilon_{a}$ & 0.36 & 0.26 & 0.24 & 0.27 & 0.34 \\
$\Delta \varepsilon_{y y}^{a v g} / \varepsilon_{a}$ & 0.30 & 0.24 & 0.19 & 0.18 & 0.18 \\
$\Delta \varepsilon_{x y}^{a v g} / \varepsilon_{a}$ & 0.16 & 0.18 & 0.17 & 0.17 & 0.16 \\
\hline
\end{tabular}


consistent across the applied strain measurements except for increased values in applied strains of 2 and $10 \%$ compared to $4-8 \%$. As indicated by Figures 5 and 9 , increased $\Delta \varepsilon_{x x}^{a v g} / \varepsilon_{a}$ at small strains in grain 1 can likely be attributed to experimentally observed slip lines. At larger strains, large deviation was observed near the grain boundary between grains 1 and 4 . This discrepancy indicates a possible deficiency in the model's handling of grain boundaries. Large $\Delta \varepsilon_{x x}^{a v g} / \varepsilon_{a}$ at large strains could also be a result of the model not accounting for damage.
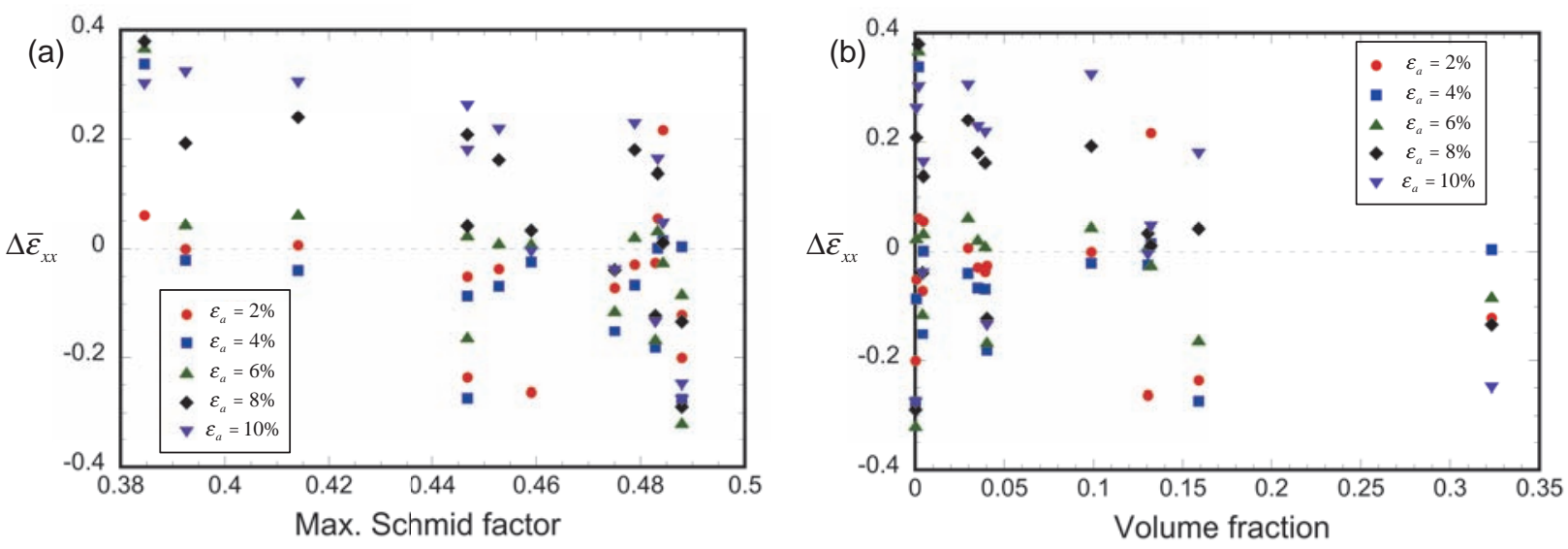

Figure 10: Calculated $\left(\varepsilon_{x x}^{\mathrm{DIC}}-\varepsilon_{x x}^{\mathrm{sim}}\right) / \varepsilon_{a}$ versus (a) the maximum Schmid factor and (b) volume fraction for each grain.

To assess the fidelity of the model prediction per grain basis, averaged $\Delta \bar{\varepsilon}_{x x}=\left(\varepsilon_{x x}^{\mathrm{DIC}}-\varepsilon_{x x}^{\mathrm{sim}}\right) / \varepsilon_{a}$ within each grain were obtained. Figures 10 (a) and (b) show calculated $\Delta \bar{\varepsilon}_{x x}$ versus the maximum Schmid factor and volume fraction, respectively. As shown in Figure 10 (a), CP-FEM model underpredicted $\varepsilon_{x x}\left(\Delta \bar{\varepsilon}_{x x}>0\right)$ for grains having low maximum Schmid factors. In particular, grains 12, 5 and 8 having lowest Schmid factors (0.385, 0.392 and 0.414, respectively) and thus representing hardest grains were under-predicted by the model. In addition, the model over-predicted $\varepsilon_{x x}$ $\left(\Delta \bar{\varepsilon}_{x x}<0\right)$ for two softest grains (grains 1 and 14). This result suggests that the CP-FEM model predictions of strain fields are less accurate in grains having extreme orientations, i.e. softest and hardest grains within the specimen. On the other hand, there was no noticeable correlation between $\Delta \bar{\varepsilon}_{x x}$ and the volume fraction (or the size) of each grain within the gage section as shown in Figure $10(\mathrm{~b})$.

Although we strive to reproduce the microstructural features of the specimen and test procedures accurately using high resolution experimental techniques and a simple-structured specimen, there still exist various limitations of the current approach. The main possible source of error between the model and simulation is an assumption on columnarity of the specimen. The oligocrystal specimen used in this work does not have a perfectly columnar grain structure and it is likely that initial crystal orientations varied through the thickness direction.

While macroscopic mechanical responses of coarse-grained oligocrystals are likely to have small effects from local grain boundary properties, it is clear that a more sophisticated treatment of grain boundaries and grain boundary-dislocation interaction would improve model predictions. This is clearly shown in Figure 9 where the model underpredicted $\varepsilon_{x x}$ near grain boundaries as compared to HR-DIC measurements. Furthermore, many material parameters used in the model are assumed to be constant and homogeneous throughout the specimen, i.e. the model does not incorporate any initial heterogeneous distribution of defects. 


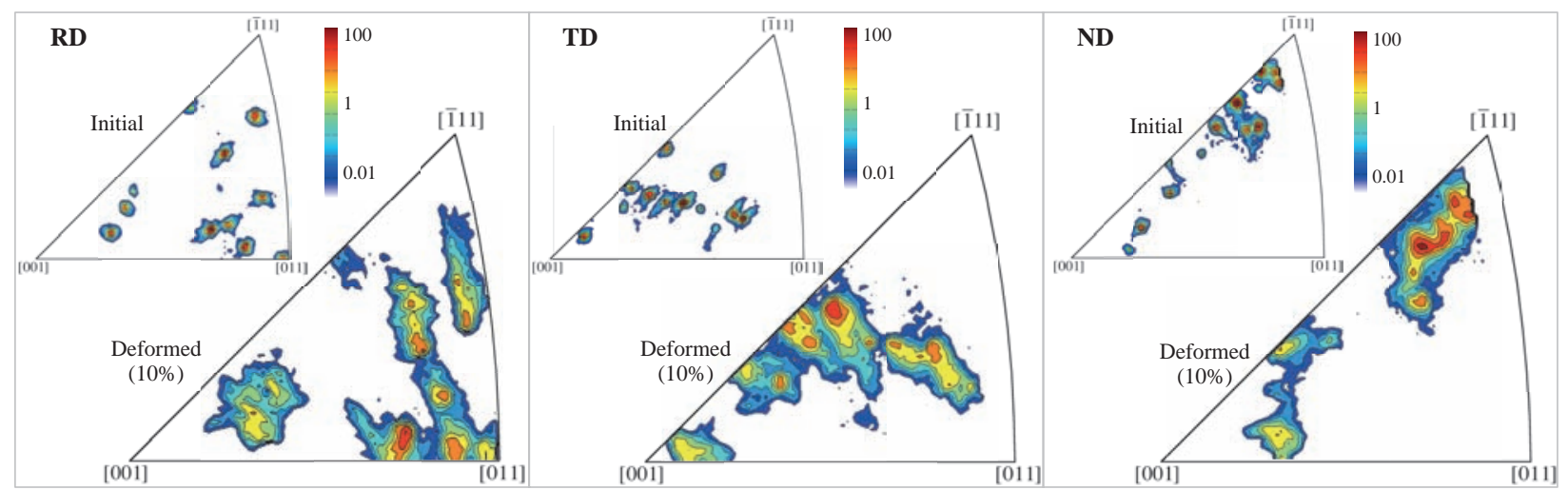

(a) Measured (EBSD)

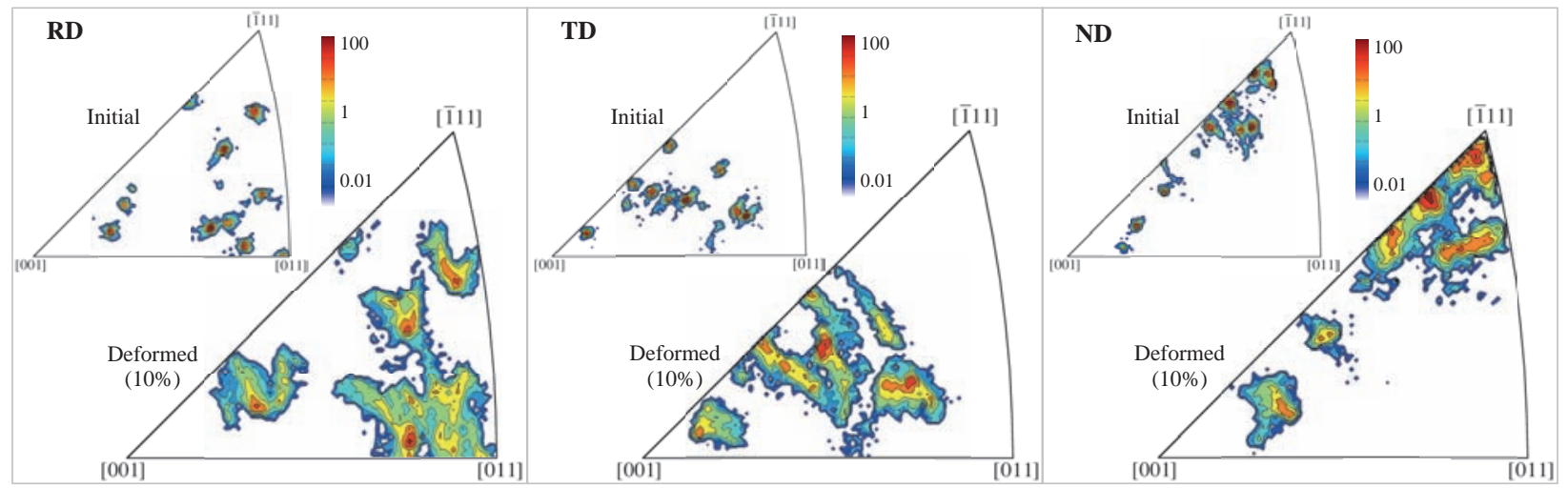

(b) Predicted (CP-FEM)

Figure 11: Comparisons of (a) measured and (b) simulated inverse pole figures in the loading direction (rolling direction), transverse direction, and normal direction, before and after deformation. The color scale represents the multiple times the random occurrence.

\subsection{Comparisons of Crystal Rotations}

In addition to measurements of surface strain fields, crystal rotations after deformation were obtained from EBSD; these measurements allowed for a comparison with CP-FEM predictions of grain rotations throughout the gage section. Figures 11 (a) and (b) show inverse pole figures of measured and simulated texture, respectively, for the initial (Point A, $\varepsilon_{a}=0 \%$ ) and the final (Point F, $\varepsilon_{a}=10 \%$ ) stages of deformation with respect to the loading/ rolling (RD), transverse (TD), and normal (ND) directions. To illustrate the texture of the specimen, an occurrence of crystal orientation data within an unit area of stereographic triangle is normalized by the random occurrence. Thus, the color scale represents the surface texture of the specimen in multiple times the random occurrence without filtering the data by the orientation distribution function (ODF).

As shown in the inverse pole figures in Figure 11, the initial crystal orientations of the oligocrystal specimen were accurately reproduced by the model. In contrast to assigning an average crystal orientation to elements within the same grain as is often done $[9,10,13,16,43,17]$, the measured spread of initial crystal orientations within grains are captured in this model. Simulated texture at 10\% deformation agrees reasonably well with the measured texture in all three directions. In particular, the model accurately predicted the spreading of the crystal orientations and paths of the crystal rotation. Observed deviations between experimental measurements and model predictions in texture can also be attributed to limitations of the model and assumptions about the speci- 


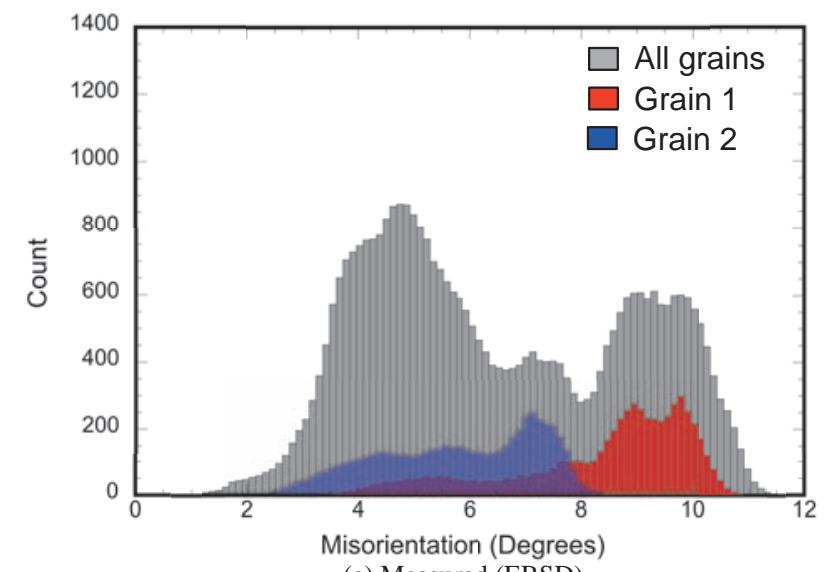

(a) Measured (EBSD)

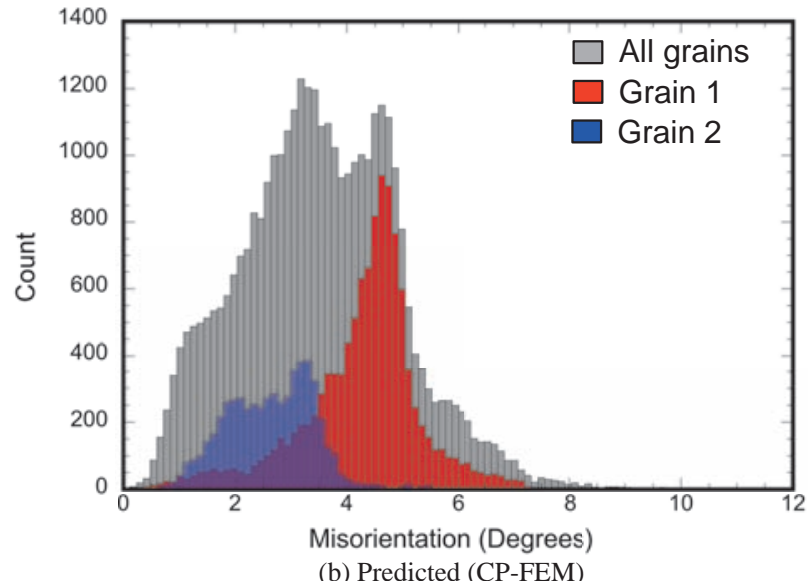

(b) Predicted (CP-FEM)

Figure 12: (a) Measured and (b) predicted distributions of misorientation angles relative to average initial crystal orientations at $10 \%$ deformation. Here, distributions of misorientation angles within the entire gage section (all grains), grain 1 and grain 2 are compared.

men, i.e. inhomogeneous crystal orientation through the thickness and a columnar grain structure. Previous work by Lim et al. [21] also showed large disagreement in crystal rotations near grain boundaries.

The representation of the crystal orientation involves three independent variables, e.g. $\phi_{1}, \Phi$, and $\phi_{2}$ for Bunge Euler angles notation. A simple scalar value to represent the magnitude of the crystal rotation can be obtained via calculating a misorientation angle relative to the initial crystal orientation. Although this use of misorientation angles neglects different orientations of the rotation axis, it allows convenient comparisons between experimental measurements and model predictions. The misorientation angle, $\theta$, between two orientations, $\mathrm{A}$ and $\mathrm{B}$, can be calculated using corresponding rotation matrices, $g^{A}$ and $g^{B}$ as follows [44]:

$$
\theta=\cos ^{-1}\left[\frac{1}{2}\left(\Delta g_{11}^{A B}+\Delta g_{22}^{A B}+\Delta g_{33}^{A B}-1\right)\right]
$$

where $\Delta g^{A B}=g^{B}\left(g^{A}\right)^{-1}$. Note that the rotation matrix, $g$, can be represented using three Bunge Euler angles, $\phi_{1}, \Phi$ and $\phi_{2}$, as follows:

$$
\begin{aligned}
g_{11} & =\cos \phi_{1} \cos \phi_{2}-\sin \phi_{1} \sin \phi_{2} \cos \Phi \\
g_{12} & =\sin \phi_{1} \cos \phi_{2}+\cos \phi_{1} \sin \phi_{2} \cos \Phi \\
g_{13} & =\sin \phi_{2} \sin \Phi \\
g_{21} & =-\cos \phi_{1} \sin \phi_{2}-\sin \phi_{1} \cos \phi_{2} \cos \Phi \\
g_{22} & =-\sin \phi_{1} \sin \phi_{2}+\cos \phi_{1} \cos \phi_{2} \cos \Phi \\
g_{23} & =\cos \phi_{2} \sin \Phi \\
g_{31} & =\sin \phi_{1} \sin \Phi \\
g_{32} & =-\cos \phi_{1} \sin \Phi \\
g_{33} & =\cos \phi_{2} \sin \Phi
\end{aligned}
$$

Figures 12 (a) and (b) show distributions of misorientation angles relative to the initial average 
orientations at $\varepsilon_{a}=10 \%$ deformation from EBSD measurements and CP-FEM simulations, respectively. Although measured and simulated distributions of $\theta$ showed similarities, i.e. two peaks observed in all grains and grain 1 showed larger $\theta$ compared to grain 2, it is also apparent that the model under-predicts the measured crystal rotations by a factor of two. Observed under-prediction of the crystal rotation by the model is consistent with the previous CP-FEM studies [10, 45, 16]. It was shown that the misorientation prediction was affected by the hardening [18] and introduction of GNDs and grain boundary effects [46]. Also note that larger spread of crystal rotations in experiments compared to simulations upon plastic deformation has been consistently observed in previous model-experiment comparison results $[10,45,16,19,21]$.

\subsection{Through-thickness Effects}

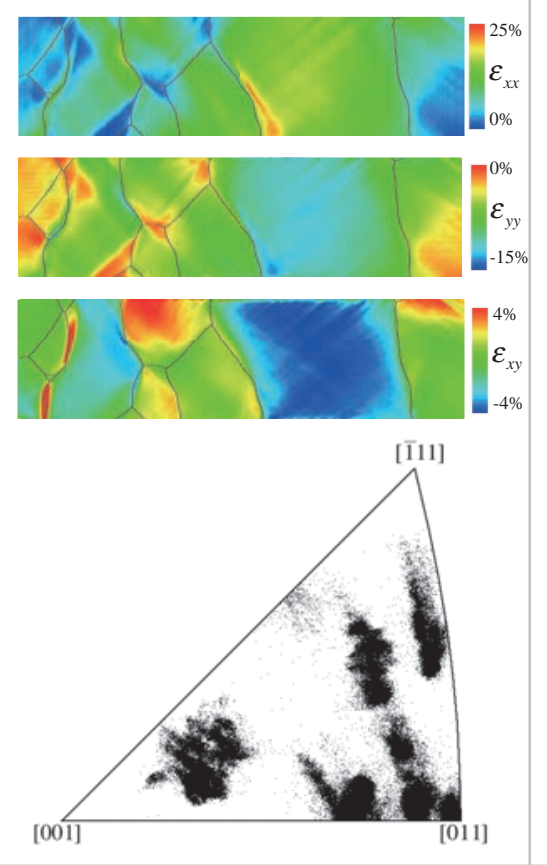

(a) Measured (Front surface)

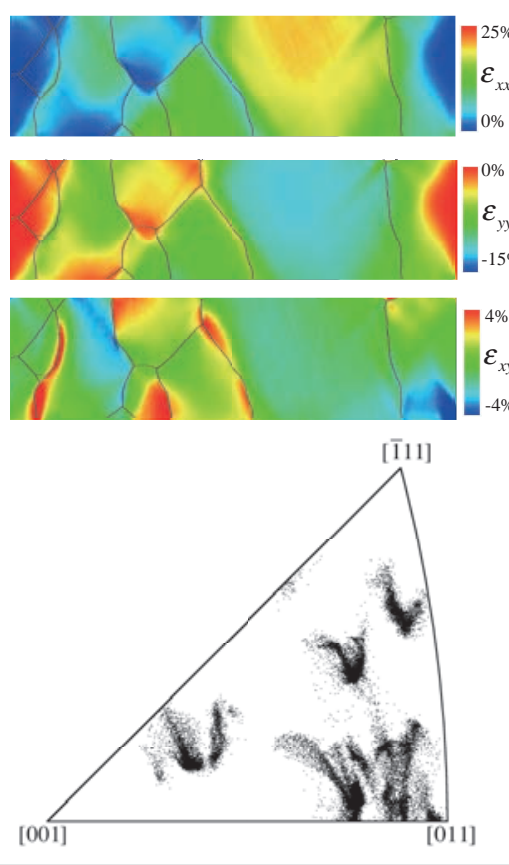

(b) CP-FEM (Front surface)
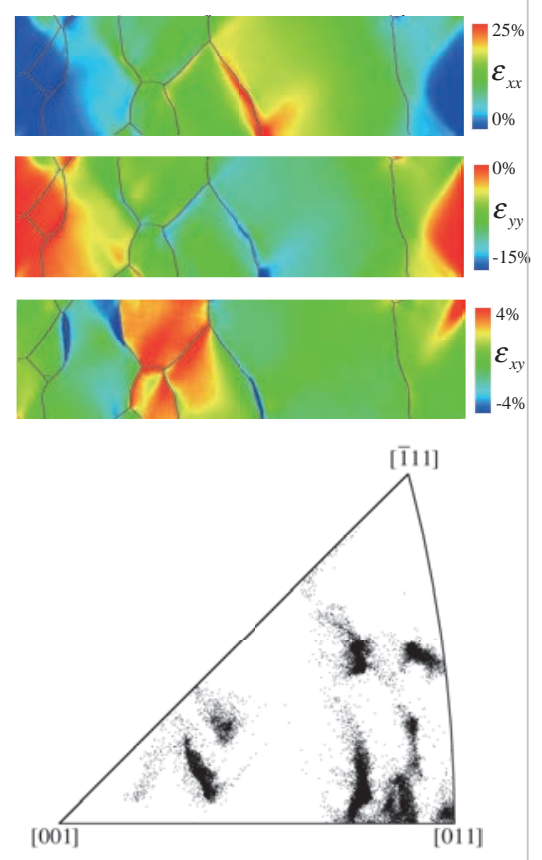

(c) CP-FEM (Back surface)

Figure 13: Measured and simulated surface strain fields and crystal orientations with respect to the loading direction at $10 \%$ deformation. (a) Measured data of the front surface, (b) simulated data of the front surface and (c) simulated data of the back surface.

The oligocrystal specimen used in this work has a relatively large thickness to width ratio $(\sim 0.6)$; thus there are significant stress and strain distributions through the thickness. These three-dimensional effects have to be considered to accurately predict surface strain fields and texture predictions. It has been shown that a full three-dimensional mesh is required to accurately model this two-dimensional structured specimen due to the three dimensional nature of the crystal orientations [21]. Figures 13 (a) - (c) compare measured and simulated surface strain and crystal orientation fields, with respect to the loading direction, at $10 \%$ deformation. Here, Figures 13 (a) and (b) show measured and simulated data on the front surface and (c) shows simulated data on the back surface. It is apparent from model predictions (Figure 13 (b) and (c)) that front and back surfaces exhibit different crystal rotations and strain fields, consistent with previous observations [21]. Note that predicted strain and crystal rotation fields on the back side do not agree with 
experimental measurements on the front side as well as front-side predictions do. One interesting feature of the back-side strain field model results is that large $\varepsilon_{x x}$ is predicted near the grain boundary between grains 1 and 4 . This location is consistent with experimentally observed $\varepsilon_{x x}$ strain localizations on the front surface of the specimen. This may suggest that the damage has initiated near the grain boundary on the back side (or subsurface).

\subsection{Effects of Hardening Laws}

In plasticity models, the choice of hardening law can have a significant effect on texture and stress-strain response predictions in single and polycrystals [47]. In addition to the dislocation density-based hardening model (Taylor hardening) [40] used in the previous sections, other commonly used formulations are isotropic hardening models [48, 49, 50, 51, 52, 53, 54] and slip-based hardening models $[1,55]$. Classical isotropic hardening models generally assume that all slip systems harden equally as a function of plastic strain. One example is the power-law hardening rule, represented as follows [48]:

$$
\tau_{\mathrm{obs}}=\tau_{0}+a_{1} \bar{\varepsilon}_{p}^{n}
$$

Here, $\tau_{0}, a_{1}$ and $n$ are material hardening parameters and $\bar{\varepsilon}_{p}$ is the equivalent plastic strain defined by,

$$
\bar{\varepsilon}_{p}=\int_{0}^{t} \sqrt{\frac{2}{3} \mathbf{F}_{\mathbf{p}}: \mathbf{F}_{\mathbf{p}}} \mathrm{d} t .
$$

Here, $\mathbf{F}_{\mathbf{p}}$ is the plastic component of the deformation gradient.

Slip-based hardening models are also widely used in crystal plasticity models and have been used to successfully predict texture and anisotropic behaviors in polycrystals $[5,6]$. In slip-based hardening models, the strain hardening term, $\tau_{\text {obs }}$, is related to the slip increment on all slip systems as follows [55]:

$$
\dot{\tau}_{\mathrm{obs}}^{\alpha}=\sum_{\beta}^{24} h^{\alpha \beta}\left|\dot{\gamma}^{\alpha}\right| .
$$

Here, $h^{\alpha \beta}$ is the hardening coefficient that relates hardening on one slip system to other active slip systems as follows [56]:

$$
h^{\alpha \beta}=\mathbf{q}^{\alpha \beta} h_{0}\left(1-\frac{g^{\beta}}{g_{s}}\right)^{a}
$$

Here, $h_{0}$ is the initial hardening rate, $g_{s}$ is the saturated flow stress and $a$ is the hardening exponent. $\mathbf{q}^{\alpha \beta}$ is a hardening matrix that determines the self to latent hardening ratios. The diagonal terms of $\mathbf{q}^{\alpha \beta}$, denoted $q_{\text {self }}$, describe self hardening while the non-diagonal terms, $q_{\text {lat }}$, describe the latent hardening effect. $q_{\text {self }}=1$ and $q_{\text {lat }}=1.4$ are the most commonly used values polycrystalline simulations $[1,5,57]$.

To investigate the effects of different hardening models on surface strain and crystal rotation field predictions in oligocrystals, CP-FEM models adopting power-law (Equation (14)) and slipbased (Equation (16)) hardening laws were fit to measured stress-strain data as shown in Figure 14. Note that the dislocation density-based, slip-based and power-law hardening laws require fitting three, four and three hardening parameters, respectively. The slip-based model has the ability to consider additional fitting parameters, i.e. $q_{\text {lat }}$ and $q_{\text {self }}$ that determine the self to latent hardening 


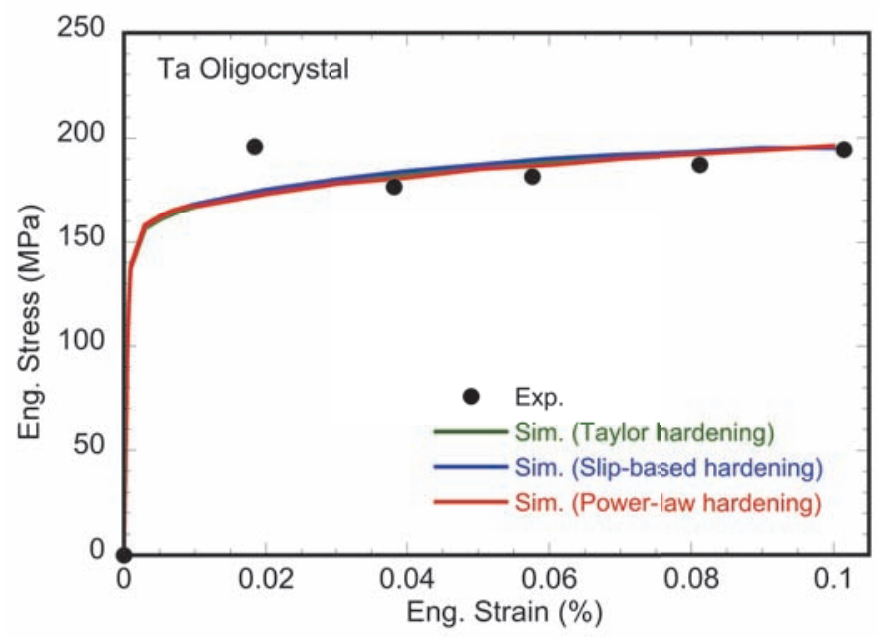

Figure 14: Measured and fitted stress-strain data of tantalum oligocrystal using three hardening laws; Taylor hardening, slip-based hardening and power-law hardening laws.

Table 4: The best-fit hardening parameters for power-law hardening and slip-based hardening models.

\begin{tabular}{cc|cc}
\hline \multicolumn{2}{c|}{ Power-law hardening } & \multicolumn{2}{c}{ Slip-based hardening } \\
\hline$\tau_{0}$ & $27 \mathrm{MPa}$ & $g_{0}$ & $27 \mathrm{MPa}$ \\
$a_{1}$ & 0.25 & $h_{0}$ & $20 \mathrm{MPa}$ \\
$n$ & 0.80 & $g_{s}$ & $100 \mathrm{MPa}$ \\
& & $a$ & 1.5 \\
\hline
\end{tabular}

ratio. CP-FEM simulations using each of the three hardening laws reproduced almost identical hardening curves in the macroscale stress-strain response of the tantalum oligocrystal as shown in Figure 14; still, these different formulations can have a noticeable impact on predictions of local stress and strain fields in polycrystals as well as in single crystals.

Figures 15 (a)-(c) compare simulated surface strain fields and inverse pole figures, with respect to the loading direction, at $10 \%$ applied strain using the three different hardening laws. It is observed that at $10 \%$ deformation, the choice of hardening model had relatively moderate effect on both texture and strain fields predictions of the oligocrystal. All three CP-FEM results using different hardening laws agreed reasonably well with experimentally measured data. The most noticeable difference in $\varepsilon_{x x}$ predictions between different models were observed in grain 1. CPFEM predictions using Taylor hardening showed larger strain localization in grain 1 while more homogeneous strain fields were predicted with isotropic power-law hardening. The average values over five applied strains of $\Delta \varepsilon_{x x}^{a v g} / \varepsilon_{a}$ are $0.29,0.31$ and 0.29 for Taylor, slip-based, and powerlaw hardening laws, respectively. These values suggest that none of the hardening models agreed significantly better than the others for this oligocrystal simulation. It has previously been shown that the choice of the hardening law has a larger impact on models for single crystal deformation, and that the effect of hardening laws become less critical with a large aggregate of grains [47].

In addition to the choice of hardening laws, other constitutive effects exist in BCC models such as the non-Schmid effect and the choice of the operating slip systems. However, it has been shown that non-Schmid effects are insignificant at room temperature for tantalum [27] and that the choice of the slip system also has a modest effect on model predictions [21]. In addition to these 


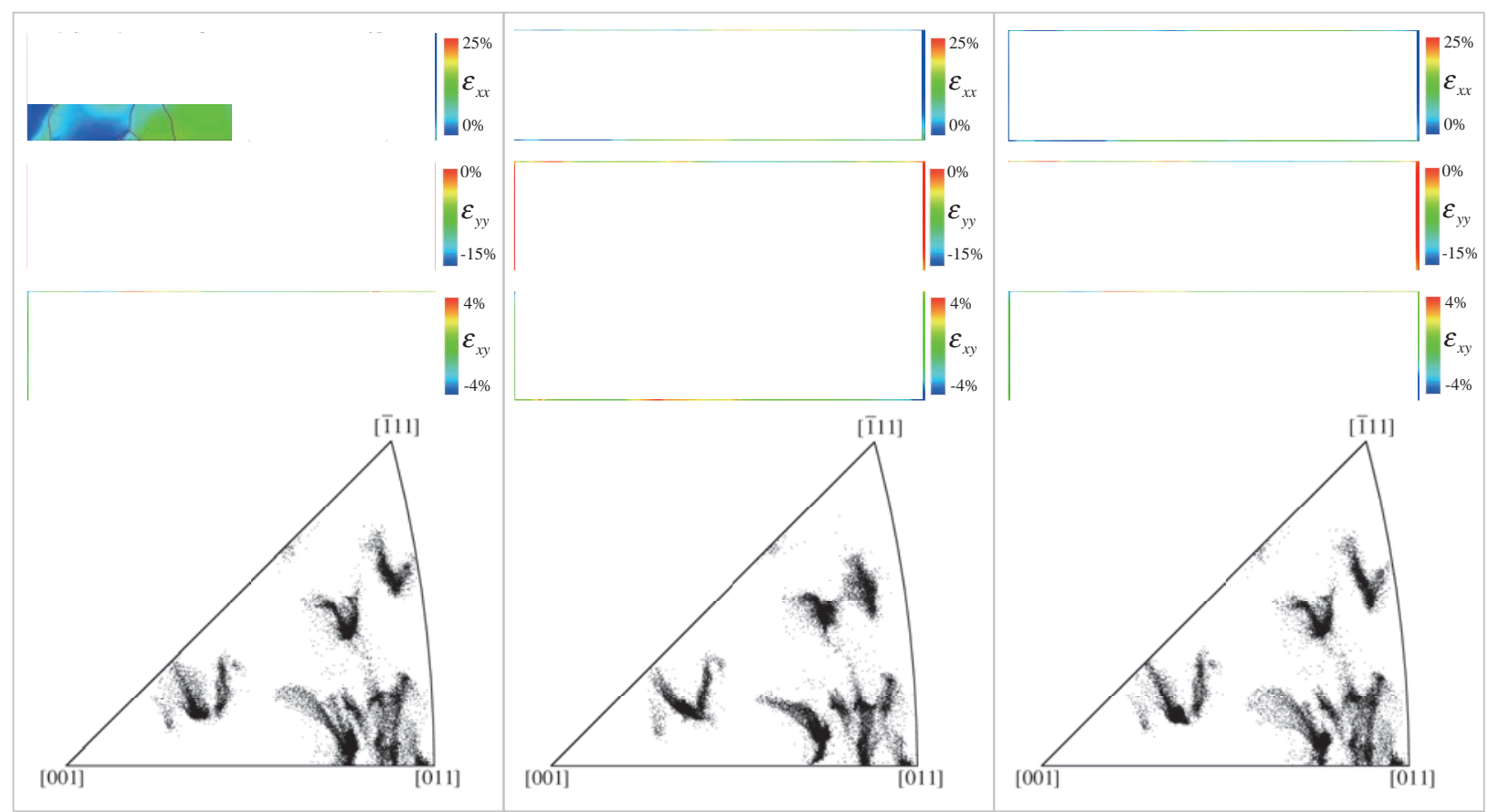

(a) Taylor hardening

(b) Slip-based hardening

(b) Power-law hardening 